\title{
Topic Study Group No. 9: Teaching and Learning of Measurement (Focus on Primary Education)
}

\author{
Christine Chambris, Barbara Dougherty, Kalyanasundaram (Ravi) \\ Subramaniam, Silke Ruwisch and Insook Chung
}

\section{The Programme}

\section{Preparation}

Measurement, the topic of this TSG, links not only to everyday contexts and application areas such as engineering, but also with other mathematical topics including number and algebraic thinking. Weak knowledge related to measurement concepts and skills often becomes problematic while studying other subjects. Yet, paradoxically, there is a lack of attention to measurement instruction in primary mathematics education internationally.

The main purpose of the TSG was to better understand conditions and constraints on the teaching and learning of measurement in international contexts, and to consider some possible changes. Specific questions announced as foci of the TSG were the extent to which

(a) measurement could be a topic in and of itself in primary school mathematics;

(b) measurement could be a vehicle for connecting other mathematical topics such as number, operations, algebra, statistics, or geometry;

Co-chairs: Christine Chambris, Barbara Dougherty.

Team members: Kalyanasundaram (Ravi) Subramaniam, Silke Ruwisch, Insook Chung.

C. Chambris ( $\square)$

Université Paris Diderot, Paris, France

and

Université de Cergy-Pontoise, Cergy-Pontoise, France

e-mail: christine.chambris@u-cergy.fr

B. Dougherty

University of Missouri, Missouri, USA

e-mail: doughertyb@missouri.edu; barbdougherty32@icloud.com

(C) The Author(s) 2017

G. Kaiser (ed.), Proceedings of the 13th International Congress on Mathematical

Education, ICME-13 Monographs, DOI 10.1007/978-3-319-62597-3_36 
(c) measurement learning could be supported by mathematical topics such as number, operations, algebra, statistics, or geometry; and

(d) informal knowledge of and conceptual understanding about measurement (including estimation and knowing how to use some instruments) support or hinder rich teaching and learning of measurement or of other mathematical subjects in school.

The TSG received 18 papers and one poster as submissions including 4 invited presentations from leading researchers, of which nine papers were accepted for long presentations (20 min presentation and $10 \mathrm{~min}$ discussion), nine papers for two short oral presentations sessions and one poster. Due to cancellations, the final program of TSG-9 consisted of nine long presentations and eight short presentations.

In relation with the four questions, four major themes emerged and later structured the four sessions: connections between measurement and other mathematical topics, connections between measurement and everyday life, how conceptual understanding of measurement of geometrical quantities develops, connections between math and physics through measurement. From the short oral presentations; a fifth theme emerged: estimation.

\section{Implementation}

\section{Day 1 (July 26)}

In the main opening session, welcome and introductions were done by Christine Chambris (co-chair) and Silke Ruwisch (team member). In her introductory remarks, Christine Chambris recalled the key issues discussed in the TSG of ICME 12, and referred to the paradox of the lack of attention to measurement in mathematics education referred to above. The two first presentations were chosen for showing how school measurement can on the one hand foster the teaching of other mathematical topics, and on the other hand how it can be connected with everyday life.

First, TSG-invitee Jeffrey E. Barrett (and colleagues) from the United States investigated the intersection of spatial measurement and school mathematics. They featured a research program that connects measurement to other topics in mathematics through tasks involving the coordination of number and space across representations, comparative reasoning about quantity and equivalence, and ideas about units, including grouping and partitioning. Second, Arindam Bose and K. (Ravi) Subramaniam from India showed that school-going children from low income urban households in developing countries often have work related experience through their participation in the informal economy; and how such experience gives rise to measurement related knowledge, which is different from the scientific knowledge of measurement that forms part of the curriculum. They characterized these differences and explored pedagogical implications. 
Two sessions consisting of short oral presentations took place. The first session focused on estimation (area, volume and assessment). The second session included presentations on measurement of length, area including the use of "bissemis", and time.

\section{Day 2 (July 27)}

The next main session focused on the extent to which measurement can be used as a vehicle for connecting and linking other mathematical topics (such as number, operations, algebra, statistics, or geometry). The converse issue was also addressed how mathematical topics such as number, operations, algebra, statistics, or geometry can support the development of measurement concepts in school.

Three papers were presented in this session followed by insightful discussions. First, TSG-invitee Richard Lehrer (and colleagues) from the United States presented a research project within which geometry and measurement learning was built on informal knowledge through embodied experiences of motion. Based on the teaching of geometrical measurement as a conceptual system, it showed transitions in teachers' pedagogical practices and conceptions of measurement that support children's conceptual change. This presentation suggested that a whole group of teachers can progressively but dramatically change when they are trained and supported with resources within a scientifically coherent project.

Second, Linda Venenciano from the United States presented how measurement can be used as a vehicle for developing algebraic thinking. Based on the works of El'konin and Davydov, mathematics representations (e.g., models and equations) embody quantities and their relationships and scaffold the development of reasoning skills found to be critical to successful preparation for a first course in high school algebra.

Third, Christine Chambris presented a brief history of the relations between numbers and quantities in the primary curriculum in France. She reported the changes that occurred in the French curriculum surrounding the New Math reform which resulted in the elimination of measurement from the theoretical foundations of numbers, and how such changes still impact the teaching of both numbers and measurement. This gave the participants an opportunity to reflect on the role of the New Math in their own countries.

\section{Day 3 (July 29)}

Three papers were presented in this session and thought-provoking issues were raised. First, the TSG-invitee Julie Sarama (and colleagues) from the United States presented the development of foundational cognitions and concepts of measurement in the early years framed through the research construct of a learning trajectory for length. They described how conceptual understanding of length measurement develops through a typical curriculum in the USA among children of 4-8 years of 
age. They focused on a case study of an 8-year-old who could successfully use measurement tools such as a ruler and feet-steps but struggled with strips.

Second, Jeenath Rahaman (and colleague) from India presented two classroom episodes on the construction of the concept of area measurement in India, with students in grades 6 and 7. She first explored the structure of the argumentation and showed that students' and teacher's argumentations were based on different implicit assumptions. Such analysis highlighed tensions when students move between spatial and numerical representations.

Third, Cheryl L. Eames (and colleagues) from the United States presented the evaluation of a hypothetical learning trajectory for length measurement using a partial credit Rasch model with students from grade 4-10. Samples of students' responses were presented, and the analysis highlighted the need for a means to distinguish between density of length, and density of numbers in evaluating students' knowledge.

Much intense discussion linking the ideas contained in the presentations took place in this session: learning trajectories, relations between the development of numbers and quantities, and quantitative equivalences between geometrical shapes.

\section{Day 4 (July 30)}

The fourth session consisted of one presentation followed by a review of all the TSG presentations. The TSG-invitee Valérie Munier (and Aurélie Chesnais) from France presented epistemological issues and treatment within French textbooks of measure and measurement in physics and mathematics. The presentation notably raised critical issues on modeling. Indeed, differences in how models are validated in mathematics and physics are often ignored in the teaching. They also demonstrated the need of the improvement of the distinction between empirical and theoretical aspects of measure.

Ravi Subramaniam and Christine Chambris then presented insights gained from the TSG. They noted that viewed historically, everyday measurement has increasingly disappeared from textbooks over a century (Subramaniam \& Bose (ICME-12), Chambris). Two possible explanations of this disappearance emerged: first, the process of de-mathematization that characterises the interaction between mathematics and larger societal change, and second, a change within mathematics itself (the grounding of numbers in set theory), leading to a separation of measurement from arithmetic (Chambris). Reconceptualizing the place of measurement in the curriculum may need one to address both these factors.

We might look at counter-trends to demathematization as exemplified in the out-of-school knowledge that children acquire in the informal economy of the community and leverage them for school learning (Bose \& Subramaniam). We might give a more central place to estimation: understand more carefully different kinds (or meanings) of estimation, estimation strategies, and build activities around them (Ruwisch; Pizzaro, Gorgorio \& Albarracine, Huang). We might introduce activities that build an embodied understanding of quantity through motions of 
different kinds (Lehrer). We might use measurement as a context to introduce core mathematical topics such as negative integers and algebra (Venenciano: measure up curriculum), or more generally use measurement as a connecting thread (Barrett and others).

Indeed, the teaching of measurement needs to bridge the gap between informal and formal activities. The use of standardized tools and formula often hides the conceptual features of measurement; the most fragile students often struggle when working on representations, not able to see the links with the object represented (ICME 12).

Measurement as a topic is inherently integrative. But integration brings in new theoretical perspectives and theoretical issues even for the way measurement is treated within the mathematics curriculum. Especially, it raises the issue of the theoretical model of quantity (or that of measurement) which is required to build relations between quantities, measurement and numbers (Barrett, Chambris, Eames, Lehrer, Munier \& Chesnais, Rahaman, Venenciano).

\section{Reflection}

Generally speaking, TSG-9 had regular attendants who were ready to engage in rich discussion throughout the four sessions in a receptive atmosphere. The relatively small number of papers confirms that there is a lack of attention to this domain. Despite this, various and new issues were raised and the necessity of further international studies in the domain of measurement was emphasized by the participants. We hope that the topic study group dealing with measurement continues to serve as a well-recognized group of the congress.

Open Access Except where otherwise noted, this chapter is licensed under a Creative Commons Attribution 4.0 International License. To view a copy of this license, visit http://creativecommons. org/licenses/by/4.0/.

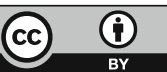

